Relato de Caso / Case Report

\title{
Macroglobulinemia de Waldenström - remissão completa após tratamento com rituximabe
}

\section{Successful outcome in Waldenström's macroglobulinemia treated with rituximab}

\author{
Flavia C. F. Pimenta ${ }^{l}$ \\ Melina P. Fernandes ${ }^{2}$ \\ Amanda R. M. Lima ${ }^{2}$ \\ Margareth F. M. Diniz ${ }^{3}$ \\ Maria Angelina C. F. Fernandes ${ }^{4}$ \\ Sandra M. C. C. Esteves ${ }^{5}$ \\ Lenísio B. de Araújo ${ }^{6}$
}

\begin{abstract}
A macroglobulinemia de Waldenström $(M W)$ é uma patologia rara dos linfócitos $B$ caracterizada pela produção monoclonal de IgM, e que pode manifestar-se clinicamente com fadiga, astenia, perda de peso, sangramento de mucosas e do trato gastrintestinal, lifonodonomegalias, hepatoesplenomegalia e alterações neurológicas. A doença é mais comum em pacientes idosos, e seus sintomas são decorrentes da hiperviscosidade sangüinea. Na MW observa-se hipergamaglobulinemia com pico monoclonal na eletroforese de proteinas séricas, niveis elevados de IgM e demais imunoglobulinas normais ou diminuídas, imunofenotipagem com linfócitos $B \mathrm{CD} 19+, \mathrm{CD} 20+e$ CD24+, aspirado de medula óssea hipercelular, e biópsia de medula óssea hipercelular com infiltração difusa de linfócitos, linfócitos plasmocitóides e plasmócitos. Atualmente, anticorpos monoclonais estão sendo usados na terapêtica da MW com grande sucesso. O rituximabe, anticorpo monoclonal anti-CD20, tem mostrado excelentes resultados no tratamento da $\mathrm{MW}$, inclusive naqueles individuos que não obtiveram resposta adequada ao tratamento convencional. Nós reportamos o caso de uma mulher de 78 anos de idade com história de fadiga, astenia, anorexia, sonolência, inquietação, urticária, dificuldade para deambular e perda excessiva de peso, aproximadamente $22 \mathrm{~kg}$ em um período de cinco meses, cujo tratamento foi realizado com rituximabe. O objetivo deste relato é apresentar uma paciente com diagnóstico de MW e revisar aspectos clínicos e terapêutico atual da doença. Rev. Bras. Hematol. Hemoter. 2008;30(5):426-429.
\end{abstract}

Palavras-chave: Macroglobulinemia de Waldenström; hipergamaglobulinemia; anticorpo monoclonal; rituximab.

\section{Introdução}

A macroglobulinemia de Waldenström (MW) é uma doença rara das células B, caracterizada pela produção monoclonal de imunoglobulinas $\mathrm{M}(\operatorname{IgM}){ }^{1}$

Afeta pessoas idosas, manifesta-se com fadiga, astenia, perda ponderal, sangramento purpúrico, linfoadenomegalia, hepatoesplenomegalia e alterações neurológicas. ${ }^{1}$

Na MW observa-se hipergamaglobulinemia, pico monoclonal na eletroforese de proteína, elevação de IgM e imunoglobulinas normais, imunofenotipagem com linfócitos B CD19+, CD20+ e CD24+, biópsia medular hipercelular com infiltração por linfócitos, linfócitos plasmocitóides e plasmócitos., ${ }^{2,3}$

${ }^{1}$ Professora da Disciplina de Hematologia da Universidade Federal da Paraiba (UFPB) - João Pessoa -PB

${ }^{2}$ Graduanda de Medicina da Universidade Federal da Paraíba (UFPB).

${ }^{3}$ Professora da Universidade Federal da Paraiba (UFPB.)

${ }^{4}$ Médica hematologista do Hospital Napoleão Laureano - HNL

${ }^{5}$ Médica cardiologista.

${ }^{6}$ Professor da Universidade Federal da Paraíba (UFPB).

Universidade Federal da Paraíba - UFPB - João Pessoa-PB

Correspondência: Flávia Cristina Fernandes Pimenta

Universidade Federal da Paraíba, Campus I, Cidade Universitária, Água Fria

58000-000 - João Pessoa-PB - Brasil

Tel: 5583 3226-5020/ Fax: 83 3221-6611

E-mail: pimenta.flavia@uol.com.br 


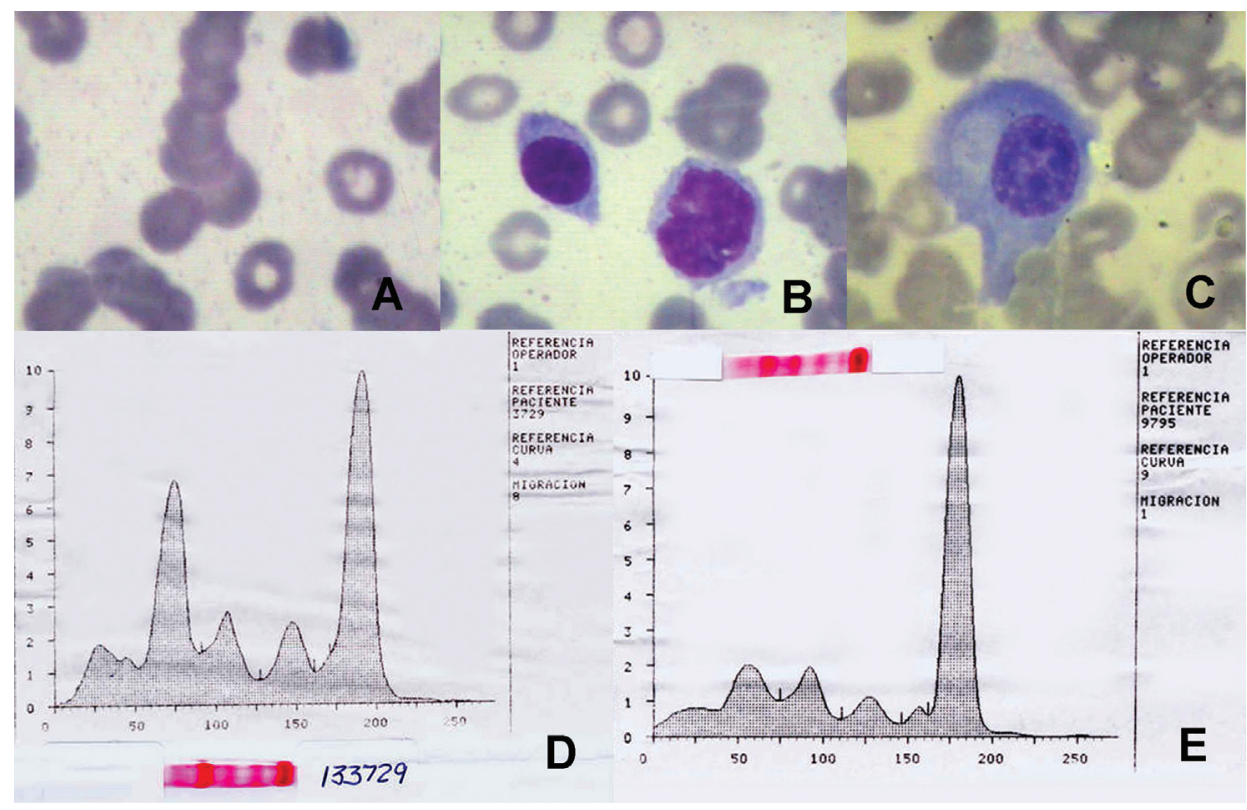

Figura 1 - A - Rouleaux eritrocitário; B - Esfregaço de sangue periférico;C - Esfregaço de medula óssea; D - Eletroforese de proteínas séricas pré-tratamento; E - Eletroforese de proteínas séricas pós-tratamento

O tratamento da MW está reservado à presença de sintomas e complicações. A plamaférese, com redução dos níveis circulantes de IgM, e a quimioterapia, com inibição do crescimento tumoral, são indicados na MW sintomática. ${ }^{1}$

A idade e a presença de citopenias estão relacionadas, significatiamente, com fatores de sobrevida. ${ }^{4}$ A sobrevida média está entre cinco e dez anos. ${ }^{5}$

\section{Relato de Caso}

T.P.S, sexo feminino, 78 anos, natural e procedente de João Pessoa - PB, encaminhada ao setor de Hematologia do Hospital Napoleão Laureano (HNL) em junho de 2006 com história de fadiga, astenia, anorexia, sonolência, inquietação, dificuldade para deambular e perda excessiva ponderal, $22 \mathrm{~kg}$ em cinco meses. Tratada como estado depressivo, não apresentava melhora sintomática, tendo realizado hemograma com evidência de leucocitose, motivo que levou familiares a procurar assistência especializada.

Exame físico: apresentava estado geral bom, orientada, consciente, hipocorada $(+/ 4+)$, eupneica, hidratada, anictérica, acianótica e afebril. Exame respiratório e cardiovascular sem alterações; abdome: baço palpável a $10 \mathrm{~cm}$ do rebordo costal esquerdo confirmado posteriormente pela ultra-sonografia de abdome superior. Não apresentava déficits neurológicos e adenomegalias.

Esfregaço de sangue periférico evidenciou rouleaux eritrocitário (imagem A) e atipia linfocitária (imagem B) posteriormente hemograma revelou: $\mathrm{Hto}=32,0 \% ; \mathrm{Hb}=10,4 \mathrm{mg} / \mathrm{dl}$, leucometria: $14.300(033 \% 0 \% 40 \% 26 \% 1 \%$ ) e plaquetometria $258.000 / \mathrm{mm}^{3}$. Análise bioquímica normal, exceto a eletroforese de proteínas séricas, que revelava hipergamaglobulinemia, pico monoclonal (imagem D), que posteriormente observouse tratar de imunoglobulina $\mathrm{M}(\operatorname{IgM})$. O diagnóstico definitivo foi feito pelo mielograma (imagem C), dosagem de IgM e imunofenotipagem. Em relação à dosagem das imunoglobulinas, observou-se IgM elevado, provas de função hepática e renal normais. Outros exames complementares foram solicitados, onde observou-se elevação da DHL (602 U/L), da $\beta 2$ microglobulina $(6,4 \mathrm{ng} / \mathrm{ml})$ e do VHS $(59 \mathrm{~mm} / \mathrm{h})$, banda monoclonal detectada a anti IgM e anti-Lambda à imunoeletroforese das proteínas.

Exame oftalmológico impossibilitado pela presença de catarata.

Desenvolveu quadro de desorientação, agitação e incoordenação motora, recebendo diagnóstico de distúrbio metabólico. Solicitada internação com hidratação adequada e instituição do tratamento escolhido após internação. Tratamento com rituximabe $375 \mathrm{mg} / \mathrm{m}^{2}$, semanalmente, durante quatro semanas. Paciente evoluiu sem intercorrências, com melhora dos sintomas. Eletroforese de proteínas séricas (póstratamento) de 31 de julho de 2006 encontrava-se dentro da normalidade (imagem E).

A continuidade do tratamento foi feita com rituximabe a cada três meses como preconizado para linfomas não-Hodgkin CD-20 positivo, por dois anos. A paciente encontra-se assintomática com todos os controles laboratoriais normais.

\section{Discussão}

A MW é uma neoplasia rara, caracterizada pela produção de clone de IgM por células B. ${ }^{20}$ 
Os pacientes se queixam de fadiga, astenia e perda de peso. Também podem manifestar sangramento, principalmente epistaxe. ${ }^{20}$ Os pacientes podem também apresentar sinais e sintomas da síndrome da hiperviscosidade. São comumente observadas linfoadenopatia e hepatoesplenomegalia ao exame físico.

A combinação da morfologia da medula óssea, histologia e imunofenotipagem distingue a MW de outras doenças das células B, como: leucemia linfocítica crônica, linfoma das células do manto, linfoma folicular, linfoma difuso de grandes células B e linfoma de zona marginal extranodal, que podem estar associadas com paraproteína IgM. ${ }^{20}$

A decisão de iniciar a terapia é apropriada para os pacientes que se apresentam com sintomas e sinais da doença. A iniciação da terapia não deve ser baseada em níveis séricos da proteína monoclonal, exceção quando a proteína monoclonal maior que $50 \mathrm{~g} / \mathrm{l}$ tem um risco elevado para síndrome de hiperviscosidade e requer observação cuidadosa em relação ao aparecimento de sangramento, turbidez visual, alteração do nível de consciência. ${ }^{11}$

O tratamento deve ser individualizado e baseado nas manifestações clínicas e potenciais efeitos tóxicos desenvolvidos pelo paciente em detrimento ao tratamento instituído. Assim, a escolha do tratamento adequado deve levar em consideração a necessidade de um rápido controle da doença, a idade do paciente e potenciais candidatos a transplante de células-tronco.

Pacientes com sintomas de hiperviscosidade devem ser tratados com plasmaférese..$^{19} \mathrm{O}$ tratamento deve ser feito concomitantemente com a quimioterapia para a redução da produção anormal de proteína IgM monoclonal.

As três principais escolhas para tratamento de pacientes sintomáticos incluem: agentes alquilantes (clorambucil), análogos nucleosídicos (cludarabina) e o anticorpo monoclonal (rituximabe). São estes os chamados agentes de primeira linha no tratamente da MW. Para escolha de monoterapia ou associação entre os agentes citados devem ser levados em consideração alguns importantes fatores: idade, presença de comorbidades, citopenias, necessidade de rápido controle da doença, pacientes candidatos a transplante autó$\operatorname{logo}$, etc. ${ }^{20}$

Agentes alquilantes e análogos de nucleosídeos são benéficos para muitos pacientes com MW que necessitam do tratamento. Entretanto, esses compostos podem induzir citopenias e disfunção imune celular, que predispõem a infecções oportunistas. ${ }^{6,7}$ Além disso, têm atividade limitada no tratamento de pacientes que apresentaram resistência primária ou secundária aos agentes quimioterápicos. Esses agentes encontram indicação quando um rápido controle da progressão da doença é necessário. ${ }^{20}$

Uma nova opção no tratamento dos pacientes com MW tem sido a administração de anticorpos monoclonais direcionados a antígenos expressos na superfície das células malignas. O rituximabe, anticorpo monoclonal anti-CD20, administrado na dose de $375 \mathrm{mg} / \mathrm{m}^{2}$ semanalmente, está tendo excelentes resultados na MW, pois pacientes com MW têm o antígeno CD20 expresso em suas células linfoplasmáticas. $^{8}$

Rituximabe é um tratamento bem-tolerado, ${ }^{20}$ pode também representar o tratamento de escolha para pacientes citopênicos, já que possui um menor potencial imunossupressor. ${ }^{11}$ Tem indicação ainda nos pacientes candidatos a transplante autólogo (alternativa à refratariedade à quimioterapia). Caso não haja necessidade de rápido controle da doença, mas haja evidência de leucopenia e trombocitopenia, o rituximabe também se faz a terapia de escolha. ${ }^{20}$ Além desses fatores mencionados anteriormente, o rituximabe foi considerado o tratamento de escolha para essa paciente em virtude da idade avançada e da possibilidade de menor efeitos adversos e maior tolerância ao tratamento quimioterápico.

Os pacientes, de maneira geral, recebem atualmente terapia adicional de acordo com sua resposta ao uso do rituximabe. Em adição, uma série de pequenos estudos restrospectivos mostrou que a monoterapia com rituximabe induz boa resposta aos pacientes com MW resistente a outras terapias, assim como demonstrou que a mesma induz boa resposta em aproximadamente $30 \%$ a $40 \%$ de pacientes previamente tratados. ${ }^{20}$

A monoterapia com rituximabe deve ser utilizada com cautela em pacientes que têm sintomas de hiperviscosidade sangüínea, pois este agente leva a um aumento transitório dos níveis séricos de IgM e, portanto, aumento abrupto da viscosidade sangüínea. ${ }^{20}$

Vários agentes estão sendo estudados para uma possível utilidade no tratamento de pacientes com $\mathrm{MW},{ }^{18}$ podem ser usados isoladamente ou em associação com outras drogas ou anticorpos monoclonais. No entanto, há dificuldade em se compararem os diversos estudos retrospectivos e prospectivos realizados acerca das novas modalidades de tratamento para MW, por ser patologia rara, incluindo assim pequeno número de pacientes nos referidos estudos, não os tornando adequadamente confiáveis. Tais agentes são requisitados para o tratamento da MW quando a doença se torna refratária às terapias de primeira linha já citadas. ${ }^{20}$

Sendo uma doença indolente, progressiva, a MW tem um curso clínico variável. Fatores associados a um pior prognóstico são: $\mathrm{Hb}<10 \mathrm{~g} / \mathrm{dL}$, idade $>65$ anos, perda de peso e crioglobulinemia. ${ }^{9,19}$ Elevado nível de $\beta 2$-microglobulina e baixo nível de albumina também estão associados a um pior prognóstico. A hiperviscosidade, anemia, hemorragia, trombose ou infecções são causas que contribuem para o óbito.

Achamos oportuno o relato do caso pela raridade do mesmo, necessidade de realizar diagnóstico diferencial com outras condições linfoproliferativas, doenças psiquiátricas e pela resposta excelente ao tratamento instituído sem efeitos adversos durante o mesmo. 


\section{Abstract}

Waldenström's macroglobulinemia is a rare pathology of $B$ lymphocytes characterized by the production of monoclonal IgM, causing clinical manifestations which may include fatigue, asthenia, weight loss, bleeding of the mucosa and intestinal tract, lymphadenomegaly, hepatosplenomegaly and neurological alterations. The disease is more frequent among elderly patients and its symptoms are a result of the hyperviscosity of blood. Waldenström's macroglobulinemia presents hypergammaglobulinemia with a monoclonal peak of serum proteins seen by electrophoresis, high IgM levels and other normal or diminished immunoglobulin levels, immunophenotyping with CD19+, CD20+ and CD24+B lymphocytes aspirated from hypercellular bone marrow and hypercellular bone marrow biopsy with diffuse infiltration of lymphocytes, plasmocytoid lymphocytes and plasmocytes. Currently, monoclonal antibodies are successfully being used in the treatment of Waldenström's macroglobulinemia. Rituximab, an anti-CD20 monoclonal antibody, has shown excellent results in the treatment of Waldenström's macroglobulinemia even for individuals who did not obtain satisfactory responses to conventional treatment. This work reports the case of a 78-yearold woman with a history of fatigue, asthenia, anorexia, somnolence, restlessness, urticaria, difficulties in walking, and excessive weight loss (approximately $22 \mathrm{Kg}$ within a period of 5 months) who was successfully treated using rituximab. The objective of this report is to present the case of this patient and to review current clinical and therapeutic aspects of the disease. Rev. Bras. Hematol. Hemoter. 2008;30(5):426-429.

Key words: Waldenström's macroglobulinemia; hypergammaglobulinemia; monoclonal antibodies; rituximab.

\section{Referências Bibliográficas}

1. Dimopoulos MA, Alexanian R. Waldenstrom's macroglobulinemia. Blood. 1994;83(6):1452-9.

2. Berger F, Isaacson PG, Piris MA et al. Lymphoplasmacytic lymphoma/ Waldenstro"m macroglobulinaemia. In: Jaffe ES, Harris NL, Stein H, Vardiman JW, eds. Pathology and Genetics Tumours ofHaematopoietic and Lymphoid Tissues. WHO OMS: IARC Press Lyon; 2001:132-134.

3. Valdez R, Finn WG, Ross CW, Singleton TP, Tworek JA, Schnitzer B. Waldenström macroglobulinemia caused by extranodal marginal zone B-cell lymphoma: a report of six cases. Am J Clin Pathol. 2001;116(5):683-90.

4. Owen RG, Barrans SL, Richards SJ et al. Waldenström macroglobulinemia. Development of diagnostic criteria and identification of prognostic factors. Am J Clin Pathol. 2001;116(3):420-8.

5. García-Sanz R, Montoto S, Torrequebrada A et al. Waldenström macroglobulinaemia: presenting features and outcome in a series with 217 cases. Br J Haematol. 2001;115(3):575-82.

6. Dimopoulos MA, Panayiotidis P, Moulopoulos LA et al. Waldenström's macroglobulinemia: clinical features, complications, and management. J Clin Oncol. 2000;18(1):214-26.

7. Kyle RA, Greipp PR, Gertz MA, et al. Waldenström's macroglobulinaemia: a prospective study comparing daily with intermittent oral chlorambucil. Br J Haematol. 2000;108(4):737-42.

8. Jensen GS, Andrews EJ, Mant MJ et al. Transitions in CD45 isoform expression indicate continuous differentiation of a monoclonal
$\mathrm{CD} 5+\mathrm{CD} 11 \mathrm{~b}+\mathrm{B}$ lineage in Waldenstrom's macroglobulinemia. Am J Hematol. 1991;37(1):20-30

9. Phekoo KJ, Jack RH, Davies E et al. The incidence and survival of Waldenström's Macroglobulinaemia in South East England. Leuk Res. 2008;32(1):55-9,

10. McLaughlin P, Grillo-López AJ, Link BK et al. Rituximab chimeric anti-CD20 monoclonal antibody therapy for relapsed indolent lymphoma: half of patients respond to a four-dose treatment program. J Clin Oncol. 1998;16(8):2825-33.

11. Dimopoulos MA, Kyle RA, Anagnostopoulos A, Treon SP. Diagnosis and management of Waldenstrom's macroglobulinemia. J Clin Oncol. 2005;23(7):1564-77.

12. Epenetos AA, Rohatiner A, Slevin M, Woothipoom W. Ankylosing spondylitis and Waldenström's macroglobulinaemia: a case report. Clin Oncol. 1980;6(1):83-4.

13. Tepper A, Moss CE. Waldenstrom's macroglobulinemia: search for occupational exposure. J Occup Med. 1994;36(2):133-6.

14. Silvestri F, Barillari G, Fanin R et al. Risk of hepatitis C virus infection, Waldenström's macroglobulinemia, and monoclonal gammopathies [letter, comment]. Blood. 1996;88(3):1125-6.

15. van Breugel HF, de Groot PG, Heethaar RM, Sixma JJ. Role of plasma viscosity in platelet adhesion. Blood. 1992;80(4):953-9.

16. Reinhart WH, Lutolf O, Nydegger UR et al. Plasmapheresis for hyperviscosity syndrome in macroglobulinemia Waldenström and multiple myeloma: influence on blood rheology and the microcirculation. J Lab Clin Med. 1992;119(1):69-76.

17. Treon SP, Agus TB, Link B et al. CD20-directed antibody-mediated immunotherapy induces responses and facilitates hematologic recovery in patients with Waldenstrom's macroglobulinemia. J Immunother. 2001;24(3):272-9.

18. Mitsiades CS, Mitsiades N, Richardson PG et al. Novel biologically based therapies for Waldenstrom's macroglobulinemia. Semin Oncol. 2003;30(2):309-12.

19. Dimopoulos MA, Kyle RA et al. Diagnosis and management of Waldenstrom's macroglobulinemia. J Clin Oncol. 2005;23(7): 1564-77.

20. Vijay A, Gertz MA. Waldenström macroglobulinemia. Blood. 2007; 109(12):5096-103.

Avaliação: Editor e dois revisores externos

Conflito de interesse: não declarado

Recebido: 25/03/2008

Aceito após modificações: 06/05/2008 